\title{
Dust in High-Velocity Clouds : relevance for Planck
}

\author{
Marc-Antoine Miville-Deschênes* \\ Institut d'Astrophysique Spatiale, Orsay, France \\ E-mail: mamdeias.u-psud.fn
}

\section{François Boulanger}

Institut d'Astrophysique Spatiale, Orsay, France

E-mail: boulanger@ias.u-psud.fr

\section{Peter G. Martin}

Canadian Institute for Theoretical Astrophysics, Toronto, Canada

E-mail: pgmartin@cita.utoronto.ca

\section{Felix Jay Lockman}

NRAO, Green Bank, WV, USA

E-mail: jlockman@nrao.edu

William T. Reach

Spitzer Science Center, Pasadena, CA, USA

E-mail: reach@ipac.caltech.edu

\section{Alberto Noriega-Crespo}

Spitzer Science Center, Pasadena, CA, USA

E-mail: alberto@ipac.caltech.edu

The recent detection of dust emission in Complex C, the largest High-Velocity Cloud (HVC) on the sky, opens a very interesting perspective for Planck. The HVC dust temperature determined using IRAS and Spitzer observations is low $(T \sim 10.7 \mathrm{~K})$ in accordance with its great distance from the Galaxy ( $>5 \mathrm{kpc}$ ). Peak column density in $30 \operatorname{arcmin}$ beam is $N_{H} \sim 10^{20} \mathrm{~cm}^{-2}$ which is typical of HVCs and similar to cirrus column density in diffuse regions. On the other hand HVCs appear to be very clumpy at smaller angular scales; several observations at the arcminute scale resolution show significant structure and higher brightness contrasts than in typical cirrus emission. In this contribution we show that, even with their moderate column density, the cold temperature, high emissivity and high column density contrast of HVCs should lead to significant and detectable emission in the Planck-HFI frequency range. In order to separate the HVC emission from the Galactic cirrus emission, the use of $21 \mathrm{~cm}$ observations will be mandatory.

$C M B$ and Physics of the Early Universe

20-22 April 2006

Ischia, Italy

\footnotetext{
*Speaker.

${ }^{\dagger}$ A footnote may follow.
} 


\section{Introduction}

High-Velocity clouds (HVC) are diffuse emission structures first detected at $21 \mathrm{~cm}$ which have average velocity forbidden by the Galactic rotation. HVCs are observed on $30 \%$ of the sky (see Fig. 1) and have angular sizes ranging from less than one square degree (Compact HVC) to 2000 square degrees for Complex C. The origin of HVCs is still a matter of debate; they could be 1) gas expelled from the Galactic disk due to star formation activity (i.e. Galactic fountain), 2) interaction with satellite galaxies - including the Magellanic clouds, 3) condensation of the coronal gas and 4) accretion of primordial gas - gas still in the Local group cluster that has never formed star. To conclude on the origin of HVCs, measurements of their distance (and therefore mass) as well as their metallicities are essential.

Several arguments are invoked to exclude the Galactic fountain scenario, including the fact that HVCs do not follow Galactic rotation and that several HVCs are too massive to have been expelled even by several supernovae; when distance estimates are available the mass of HVCs are in the $10^{5}-10^{6} \mathrm{M}_{\odot}$ range. HVCs have also been observed around other galaxies like M31 [6] where they are at distances of $<60 \mathrm{kpc}$ from the disk and have masses ranging from $10^{5}$ to $10^{7} \mathrm{M}_{\odot}$.

It is now widely considered that many of them might be infalling clouds fueling the Galaxy with low metallicity gas. This hypothesis received strong support from ultraviolet observations showing that some HVCs have a subsolar metallicity [8] and a D/H ratio compatible with primordial abundances of deuterium [5]. There is also the suggestion [1] that Compact-HVCs could be the residual of the formation of the Local group and the equivalent of the mini-dark halos which are observed in simulations of structure formation. In this scenario the largest HVCs on the sky would be some of those clouds that are falling on the Galaxy, trapped by its gravitational potential well.

The infall of fresh and metal-poor gas on the Galaxy is required to explain several important properties of Galactic ecology : the star formation rate, the constant metallicity of G stars and the presence of a bar. The exact nature of this infalling gas is still unknown. Some of it could be the HI seen in HVC but looking at external galaxies like M31, 10 to 100 more mass than what is seen in $\mathrm{HI}$ is needed. Part of this mass is most probably in the form ionized gas that continuously condense into HI but there is most likely also dark matter. Anyhow HVCs could provide essential clues in our understanding of galaxy formation and evolution.

\section{First detection of dust emission in a HVC}

Recently we [3] presented the first detection of dust emission from a HVC made by comparing sensitive Spitzer Space Telescope infrared and Green Bank Telescope $21 \mathrm{~cm}$ observations. This study focused on the Spitzer Extra-Galactic First Look Survey (XFLS) field, a diffuse HI area at high Galactic latitude, located on the edge of Complex $C$ (subsolar metallicity $(\sim 0.1-0.3)$ and distance greater than $5 \mathrm{kpc}$ from the sun [9]). Previously dust emission has been unsuccesfully looked for in HVCs using IRAS data [7]. Upper limits were found to be compatible with their low HI column densities, low metallicity (and therefore low dust/gas ratio) and their large distance (i.e. fainter radiation field). The detection of dust emission [3] has only been made possible by the use of more sensitive infrared data (Spitzer and IRIS - a reprocessing of the IRAS data [4) and higher resolution and more sensitive $21 \mathrm{~cm}$ observations (GBT). 


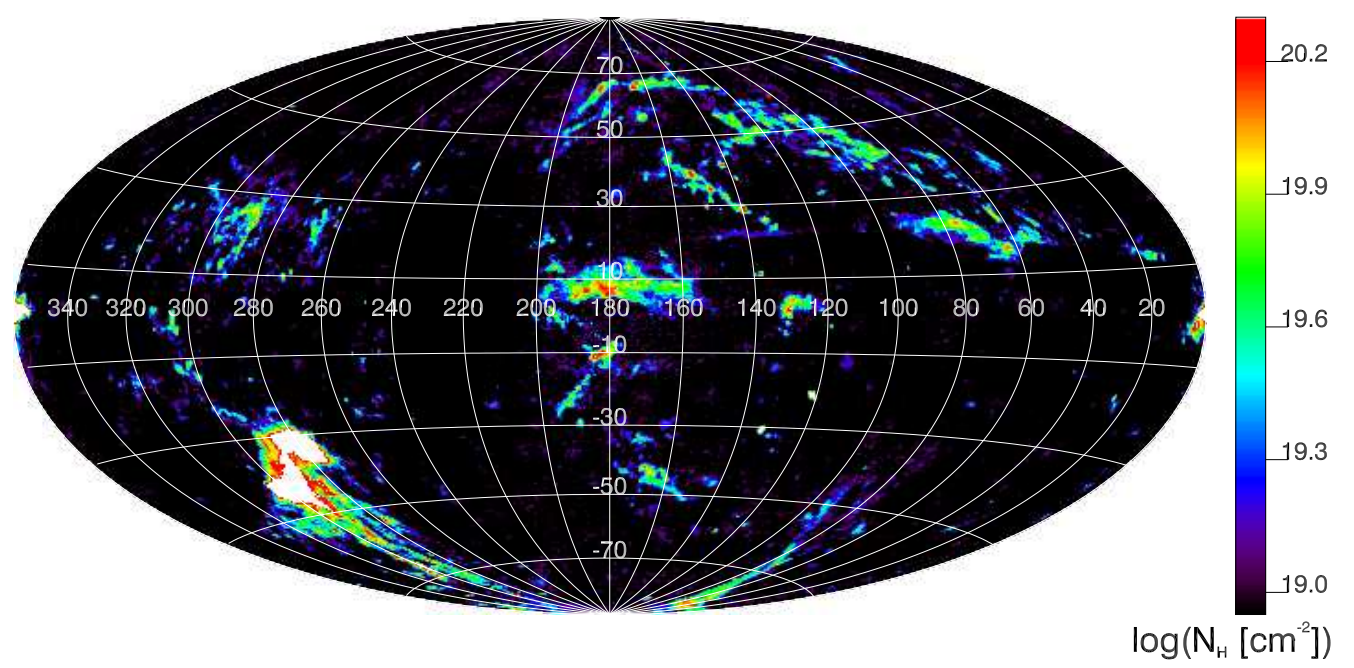

Figure 1: All-sky map of the High-Velocity Clouds (HVC) at $21 \mathrm{~cm}$ as given by the Leiden-Argentine-Bonn (LAB) survey [2]. The bright region at $(1 \sim 290, \mathrm{~b} \sim-40)$ is the Magellanic Clouds.

Dust in this HVC is found to be significantly colder than in the local interstellar medium, even at the $3 \sigma$ limit $\left(T_{B G}^{H V C}=10.7_{-3.0}^{+2.9}\right)$, which is consistant with a lower radiation field than in the Solar neighborhood due to its distance to the Galaxy [7]. In addition, from the correlation between infrared and $21 \mathrm{~cm}$ data, we report a far-infrared emissivity $\varepsilon_{H V C}$ (i.e. emission from big grains per $\mathrm{H}$ atom) higher in the HVC than in the Solar neighborhood $\left(\varepsilon_{H V C} / \varepsilon_{\text {soln }}>1.6(3 \sigma)\right)$. For dust properties typical of the local interstellar medium, $\varepsilon_{H V C}$ should be proportional to the dust-to-gas mass ratio which, for standard metal depletion on grains, would scale with the metallicity. The fact that $\varepsilon_{H V C} / \varepsilon_{s o l n}$ is greater than the metallicity in Complex $\mathrm{C}\left(Z_{H V C} / Z_{\odot}=0.2 \pm 0.1\right)$ opens interesting questions about the nature of the baryons in HVCs (see [3] for details).

\section{Can HVCs be detected with Planck?}

The detection of dust emission in Complex C shows that dust in HVCs is cold ( $T_{\text {dust }} \sim 10 \mathrm{~K}$ ) and has a higher emissivity per $N_{H I}$ than in Galactic cirrus. Using the Complex $\mathrm{C}$ numbers we estimated the emission of HVCs in the four highest frequency bands of HFI. In Fig. 目 we show the big grain thermal emission for a HI column density of $N_{H I}=10^{20} \mathrm{~cm}^{-2}$ for a cirrus and a HVC. To make a first order comparison in the Planck-HFI wavelength range between cirrus and HVC emission (for which nothing is known about the dust spectrum) we used a very simple dust model with a dust spectral index $\beta=2$. According to this simple model, dust emission from HVCs could dominate the thermal dust emission in low cirrus column density regions. This leaves no doubt about the capacity of Planck-HFI to detect the HVC thermal dust emission.

At scales larger than 30 arcmin the ratio of HVC to cirrus HI column density is $<0.5$. On the other hand observations at higher angular resolution of specific HVCs have shown that this ratio increases significantly at smaller scales. The structure of HVCs is generally more clumpy and has 


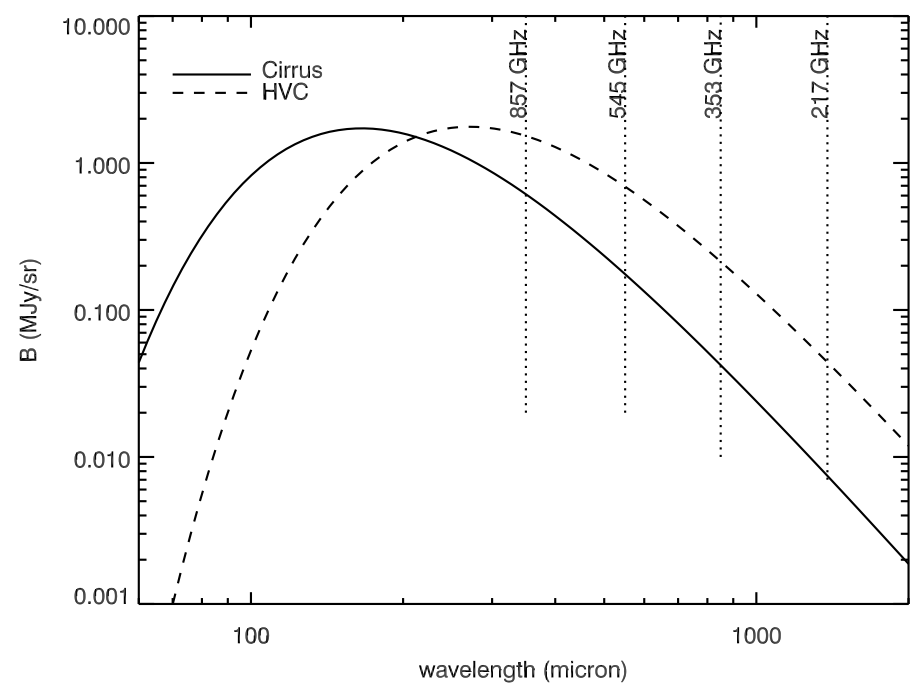

Figure 2: Comparison of thermal dust emission for a typical cirrus $\left(T_{d u s t}=17.5 \mathrm{~K}\right)$ and a $\mathrm{HVC}\left(T_{d u s t}=\right.$ $10.7 \mathrm{~K}$ ) assuming a dust spectral index $\beta=2$. The vertical dotted lines show the expected brightness sensitivity for the four highest frequencies of Planck-HFI ( $1 \sigma$ for 14 months in 5 arcmin pixel is $0.02,0.02$, 0.01 and $0.007 \mathrm{MJy} / \mathrm{sr}$ at 857, 545, 353 and $217 \mathrm{GHz}$ ).

stronger contrast than the cirrus emission. This difference in morphology should help identify and separate the HVC from the local cirrus emission in the Planck-HFI data.

\section{References}

[1] Blitz, L., Spergel, D. N., Teuben, P. J., Hartmann, D. \& Burton, W. B. High-Velocity Clouds: Building Blocks of the Local Group, 1999, ApJ, 514, 818.

[2] Kalberla, P. M. W., et al. The Leiden/Argentine/Bonn (LAB) Survey of Galactic HI. Final data release of the combined LDS and IAR surveys with improved stray-radiation corrections, 2005, A\&A, 440, 775 .

[3] Miville-Deschênes, M. A., Boulanger, F., Reach, W. T. \& Noriega-Crespo, A. The First Detection of Dust Emission in a High-Velocity Cloud, 2005, ApJ, 631, L57-L60.

[4] Miville-Deschênes, M. A. \& Lagache, G. IRIS: A New Generation of IRAS Maps 2005, ApJS, 157 , 302

[5] Sembach, K. R., et al. The Deuterium-to-Hydrogen Ratio in a Low-Metallicity Cloud Falling onto the Milky Way, 2004, ApJS, 150, 387.

[6] Thilker, D. A., et al. On the Continuing Formation of the Andromeda Galaxy: Detection of H I Clouds in the M31 Halo, 2004, ApJ, 601, L39.

[7] Wakker, B. P. \& Boulanger, F. A search for dust in high-velocity clouds, 1986, A\&A, 170, 84.

[8] Wakker, B. P., et al. Accretion of low-metallicity gas by the Milky Way, 1999, Nature, 402, 388.

[9] Wakker, B. P. Distances and Metallicities of High-and Intermediate-Velocity Clouds, 2001, ApJS, 136, 463. 